\title{
GOSAT Proto Flight Model Development
}

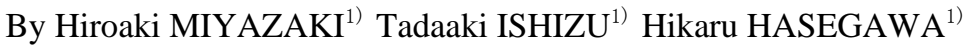 \\ and Masahiro KASUYA ${ }^{2)}$ \\ ${ }^{1)}$ Mitsubishi Electric Corporation Kamakura Works, Kamakura, Japan \\ 2) Japan Aerospace Exploration Agency, Tsukuba, Japan
}

(Received April 17th, 2008)

\begin{abstract}
Greenhouse Gases Observing Satellite (GOSAT) is under developing to launch in 2008 fiscal year. In this paper the main feature of the satellite bus system, subsystems and the development status of the bus system are presented.
\end{abstract}

Key Words: Proto Flight Model, Bus System, Structure Thermal Model, Engineering Model, System Test

\section{Introduction}

Japan Aerospace Exploration Agency (JAXA) plans to launch Greenhouse Gases Observing Satellite (GOSAT) in 2008 fiscal year. Mitsubishi Electric Corporation is developing the satellite bus system under JAXA 's leadership.

Considering the ADEOS-II solar paddle failure on orbit, several schemes are adopted for GOSAT to increase reliability. In this paper, the feature of the satellite bus system, subsystems and the development status of the satellite bus system are presented.

\section{GOSAT System}

\subsection{System characteristics}

System block diagram and main system characteristics are shown in Table1.

Table 1. System characteristics.

\begin{tabular}{|l|l|}
\hline Launch Vehicle & H-IIA \\
\hline Launch Site & $\begin{array}{l}\text { Tanegashima Space Center, } \\
\text { Kagoshima }\end{array}$ \\
\hline Orbit & $\begin{array}{c}\text { Type } \quad \text { Sun Synchronous } \\
\text { Subcurrent }\end{array}$ \\
& $\begin{array}{l}\text { Altitude }: 666 \mathrm{~km} \\
\text { Inclination }: 1.71 \mathrm{rad}\end{array}$ \\
& Period $: 5880 \mathrm{sec}$ \\
\hline Weight & $1750 \mathrm{~kg}$ \\
\hline Life & 5 years \\
\hline Mission Sensors & $\begin{array}{l}\text { Thermal And Near infrared Sensor } \\
\text { for carbon Observation } \\
\text { (TANSO-FTS) } \\
\\
\text { Cloud and Aerosol Imager } \\
\text { (TANSO-CAI) }\end{array}$ \\
\hline
\end{tabular}

\subsection{Reliable bus system}

Each subsystem of the satellite adopts the reliable method which is verified on orbit as much as possible, and by using robust design for critical subsystem, high reliability of the satellite is achieved. Especially, the failures relating power supply cause fatal damage to the satellite, survivability is increased by adopting two-power bus method and so on.

\section{Subsystems}

\subsection{Telemetry tracking command subsystem(TTC)}

TTC consists of TTC-RF and TTC-DH. It receives the command from the ground station, demodulates and distributes to each subsystem. It gathers telemetry data from each subsystem, edits, records and transmits to the ground station. It also has the autonomous function and increases the flexibility of the operation.

\subsection{Mission data handling subsystem (MDHS)}

The data from mission sensors is multiplexed by MDHS, recorded in the huge memory, and send to DT subsystem. The memory size of MDHS is 48Gbyte.

\subsection{Direct transmission subsystem (DT)}

The data from MDHS is modulated at the X-band modulator, and converted to RF signal. And it is amplified at the XSSPA and transmitted to the ground station.

\subsection{Electric power supply subsystem (EPS)}

Primary power is provided from the solar array paddles at daytime on orbit, and the power is derived from the batteries at night. EPS regulates the bus voltage and distributes the power to the components. NiCd battery is adopted because it has much flight heritage. To increase survivability two-power-bus method is adopted for GOSAT.

The power from each bus line is distributed through diode, and in case of the failure at one bus line the power is provided through another bus line.

\subsection{Paddle subsystem (PDL)}

The power needed to maintain the functions of the satellite is generated by PDL subsystem. The power needed to drive the bus subsystem is generated by one 
wing, and partial observation of the mission sensors is possible even if one paddle wing fails. PDL subsystem consists of two paddle wings, deployment mechanisms, paddle drive mechanisms. The solar array paddles are folded and attached at the side panels of the satellite by the hold and deploy mechanisms at launch phase. The two paddle wings are deployed by the ordnance controller. The length of the paddle wing is about $6 \mathrm{~m}$ from the attachment to the tip. One wing generates over $2.0 \mathrm{~kW}$ at the end of mission life at the condition that the sunlight is normal to the paddle surface.

\subsection{Attitude and orbit control subsystem (AOCS)}

AOCS consists of Attitude and Orbit Control Electronics (AOCE), attitude sensors and actuators, and performs attitude acquisition after the separation from the launch vehicle, accurate attitude control and orbit control to achieve mission requirements and solar array paddle control. The first attitude control mode is sun acquisition mode and the attitude of the satellite is controlled so that the $\mathrm{X}$-axis of the satellite points to the sun. After the sun acquisition, the attitude is controlled so that the Z-axis of the satellite points to the earth center by using Earth Sensor. In the acquisition mode ,the thrusters are used as the actuators. 4 pairs of the $1 \mathrm{~N}$ thrusters arranged at the $-\mathrm{X}$-panel of the satellite are used for the acquisition mode, and they produce torque in the three directions. At the mission operating phase Reaction Wheels (RWA) and Magnetic Torquers (MTQ) are used, and Star Sensors (STT), GPS Receiver (GPS) and Inertial Reference Unit (IRU) are used as the attitude sensors.

\subsection{Reaction control subsystem (RCS)}

RCS is a mono-propellant hydrazine blow down system. RCS consists of 2 tanks of 550mm diameter, four $20 \mathrm{~N}$ thrusters, eight $1 \mathrm{~N}$ thrusters, tubes, pressure sensors, filters and valves. The tank size is determined considering the amount of the propellant required for the mission life and de-orbit. Two $20 \mathrm{~N}$ thrusters and four $1 \mathrm{~N}$ thrusters satisfy the function required for RCS, and by having two times thrusters on board, robustness is increased against the failures. If a thruster of the four $1 \mathrm{~N}$ thrusters fail, AOCE switches the control thrusters to another four $1 \mathrm{~N}$ thrusters automatically. If the worst comes to the worst that the failure happens to the switched thrusters, by selecting the good thrusters of the eight $1 \mathrm{~N}$ thrusters the functions will be maintained.

\subsection{Thermal control subsystem (TCS)}

TCS maintains the temperature of the satellite at moderate temperature range for the each component. Thermal control is performed passively using heat pipes, MLI and OSR, and performed actively using heater controlled thermostat, and this method is verified by enough heritage.

\subsection{Integration subsystem (INT)}

INT subsystem consists of mechanical integration and electrical integration. Mechanical integration includes the brackets that support the components, the tank supports that support the RCS tanks, balance weights and volts. Electrical integration includes Power Interface Unit (PIU), Diode Box (DB), wire harness, separation switches, umbilical connectors.

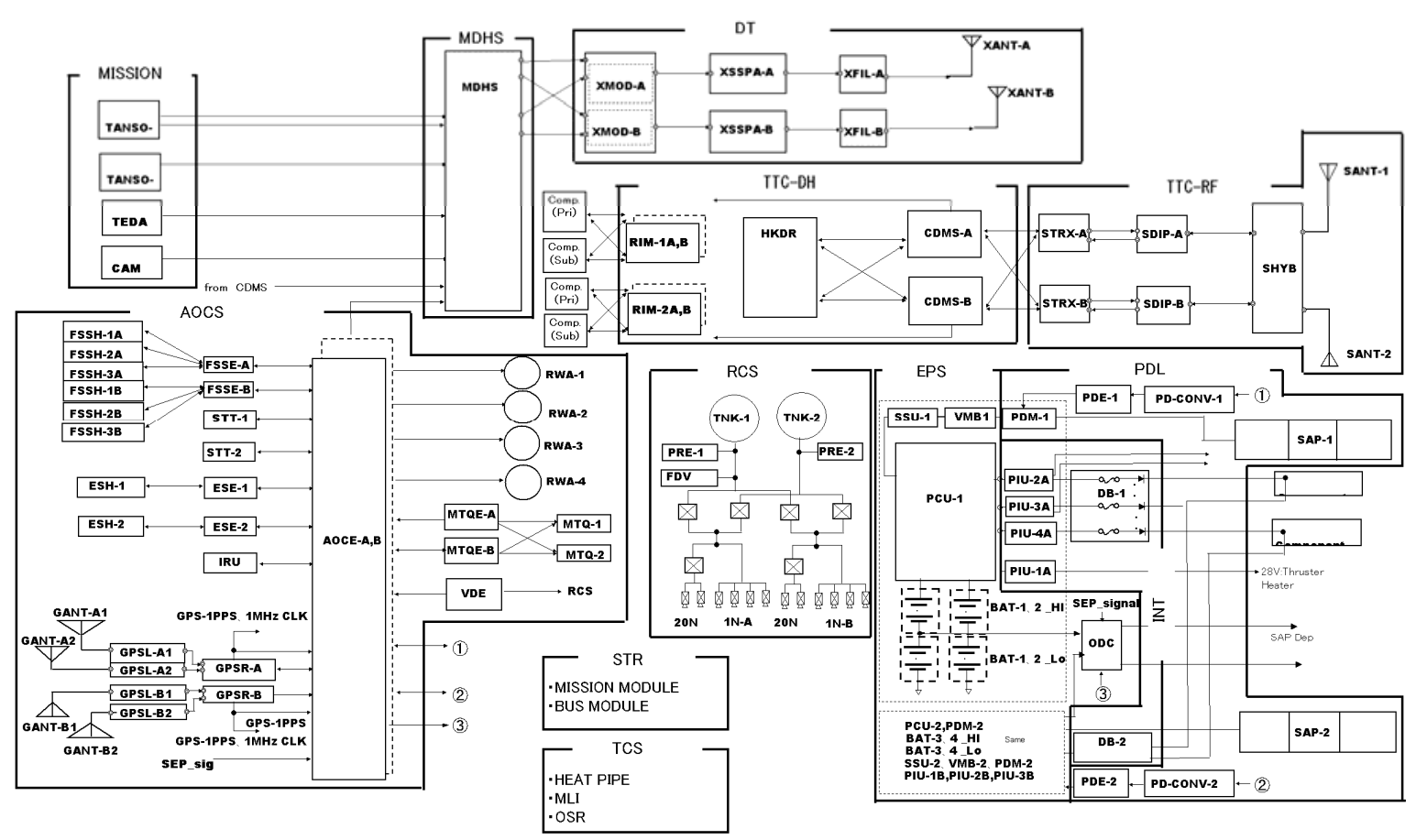

Fig. 1. System block diagram. 


\subsection{Structure (STR)}

Structure consists of the mission module that the mission sensors are loaded and the bus module that the bus components are loaded. The size is

$$
3.7 \mathrm{~m}(\text { Height }) \times 2.0 \mathrm{~m}(\text { Length }) \times 1.8 \mathrm{~m}(\text { Width }) .
$$

The mission module and the bus module can be separated so that the assembling is performed easily. There is the CFRP cylinder in the center of the bus module, and the four web support the panels that the components are loaded.

At the bottom of the bus module there is the rocket separation hardware. Two tanks of the RCS are supported inside the CFRP cylinder. The mission module consists of the honeycomb panel reinforced by CFRP on the surface. This realizes light weight and less thermal deformation.

\section{Development Status of the Satellite Bus System}

\subsection{Electrical engineering model and structure thermal model}

Before the development of the proto flight model, electrical engineering model (EEM) and structure thermal model (STM) were developed. GOSAT development policy is to use the bus components which have flight heritage as many as possible, and reduce the development risk. However, some components have to be developed, because some changes have to be done to satisfy GOSAT system requirements. CDMS, XMOD, MDP, PCU, VMB, AOCE, PIU4 and DB were developed for the electrical engineering model. After the qualification test of these EEM, system electrical test was performed using CDMS, XMOD, MDP and electrical engineering model of mission sensors to verify the interface between these components. Fig.2. shows the test scene of system electrical test. To verify mechanical and thermal interface STM was developed and several tests were performed.

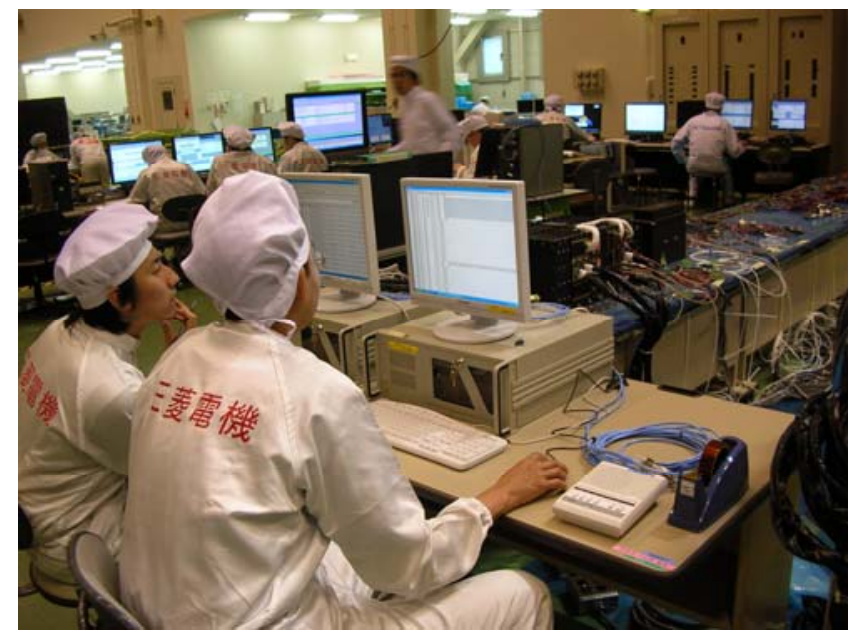

Fig.2. System electrical test (EEM).

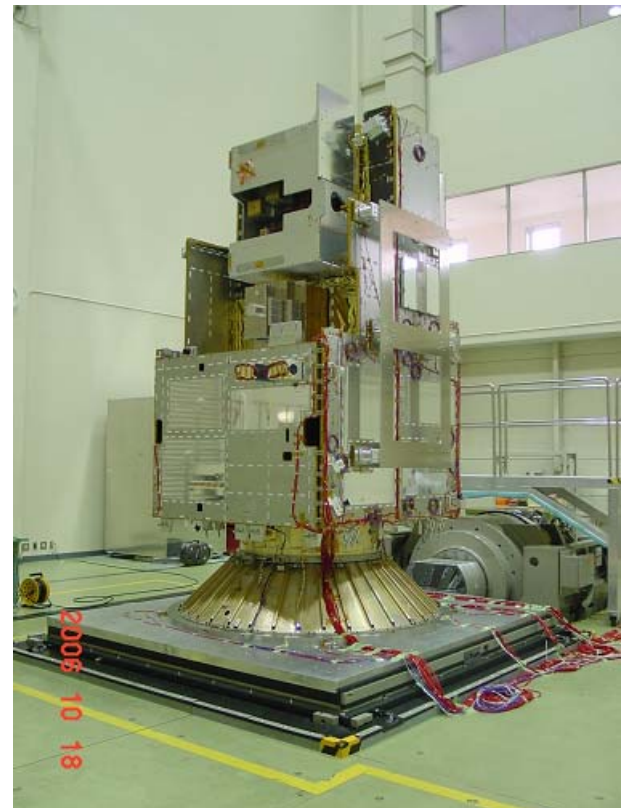

Fig.3. Vibration test (STM).

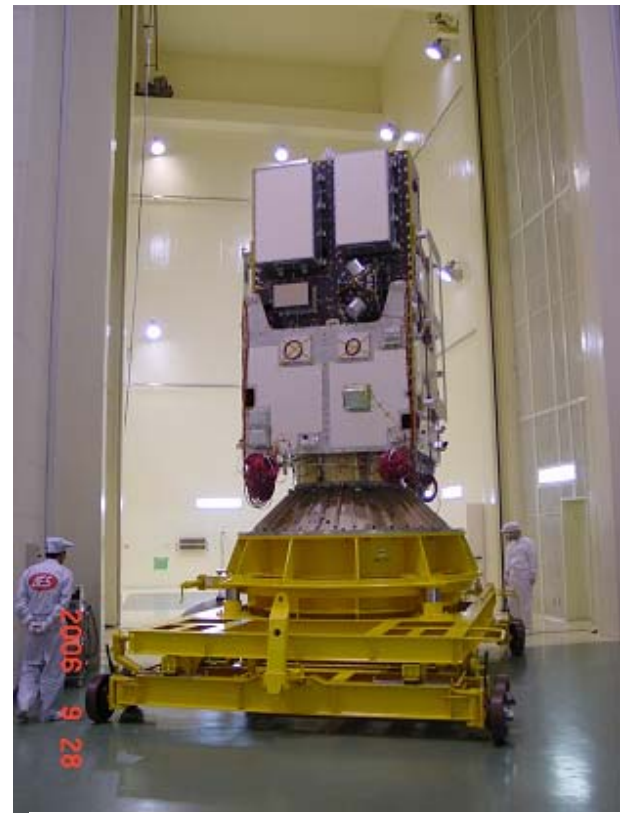

Fig.4. Acoustic test (STM).

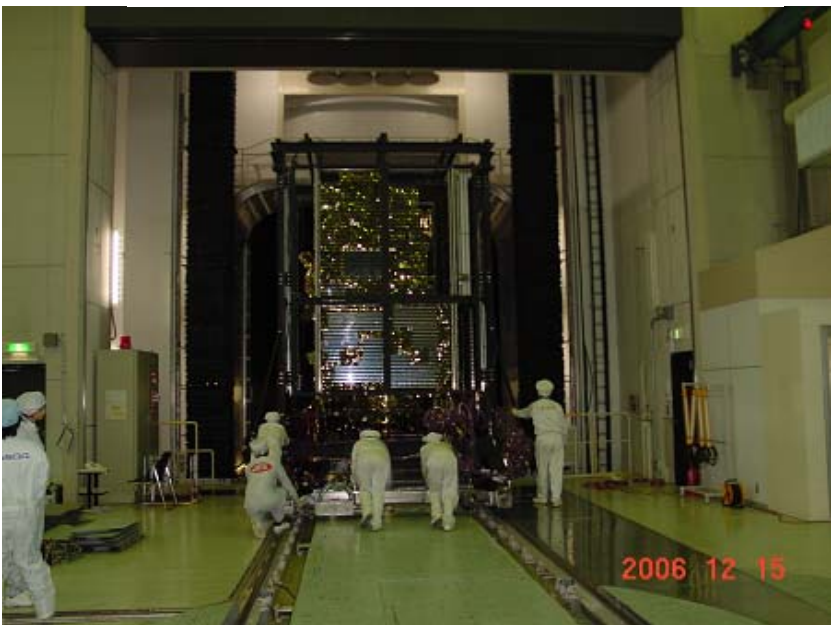

Fig.5. Thermal balance test (STM). 
Fig.3. shows the scene of vibration test. This test was performed to verify the satellite stiffness, dynamic characteristics and to establish the environmental conditions for the components.

Fig.4. shows the scene of acoustic test. This test was performed to establish environmental conditions for the components.

Fig.5. shows the scene of thermal balance test. This test was performed to verify the adequacy of thermal design and to correlate the thermal math model.

Fig.6. shows the scene of disturbance measurement test. This test was performed to verify that the in-orbit vibratory condition is low enough, as low as $3 \mathrm{mG}$, not to degrade the TANSO-FTS measurement accuracy.

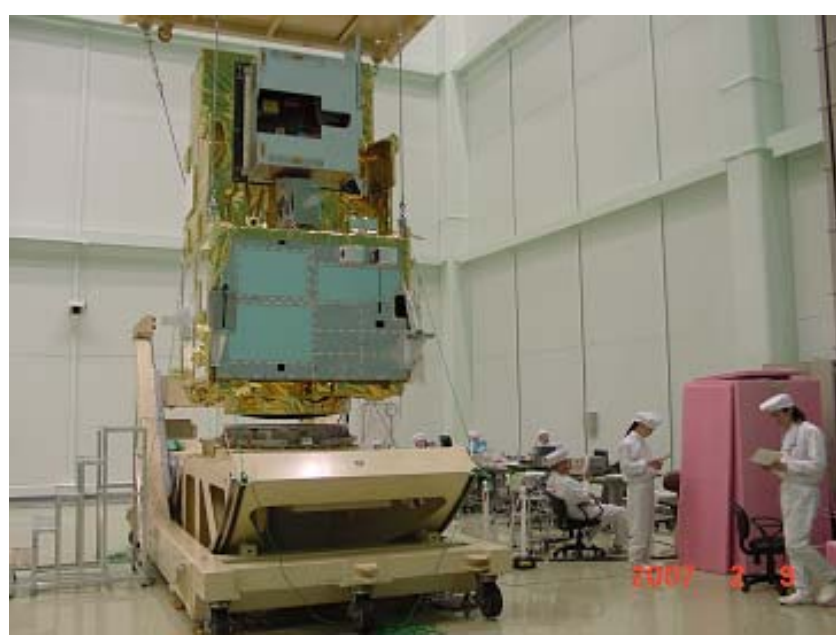

Fig.6. Disturbance measurement test (STM).

\subsection{Proto flight model}

Proto flight test for system proto flight model is now on going. All electrical components were manufactured, tested and confirmed that the functions and performance of these components satisfy the system requirements. After the proto flight test or acceptance test of these components, these components were installed to the satellite. Fig.7. and Fig.8. show the scene of the mechanical integration. After the installation of the each component, the electrical integration test is performed, and the interface, for instance power line, signal line, voltage and current between the components is verified to satisfy the interface conditions. Initial electrical performance test is performed to verify that the functions and performance of the bus system satisfy the system requirements.

Fig.9. shows the scene of system initial electrical performance test.
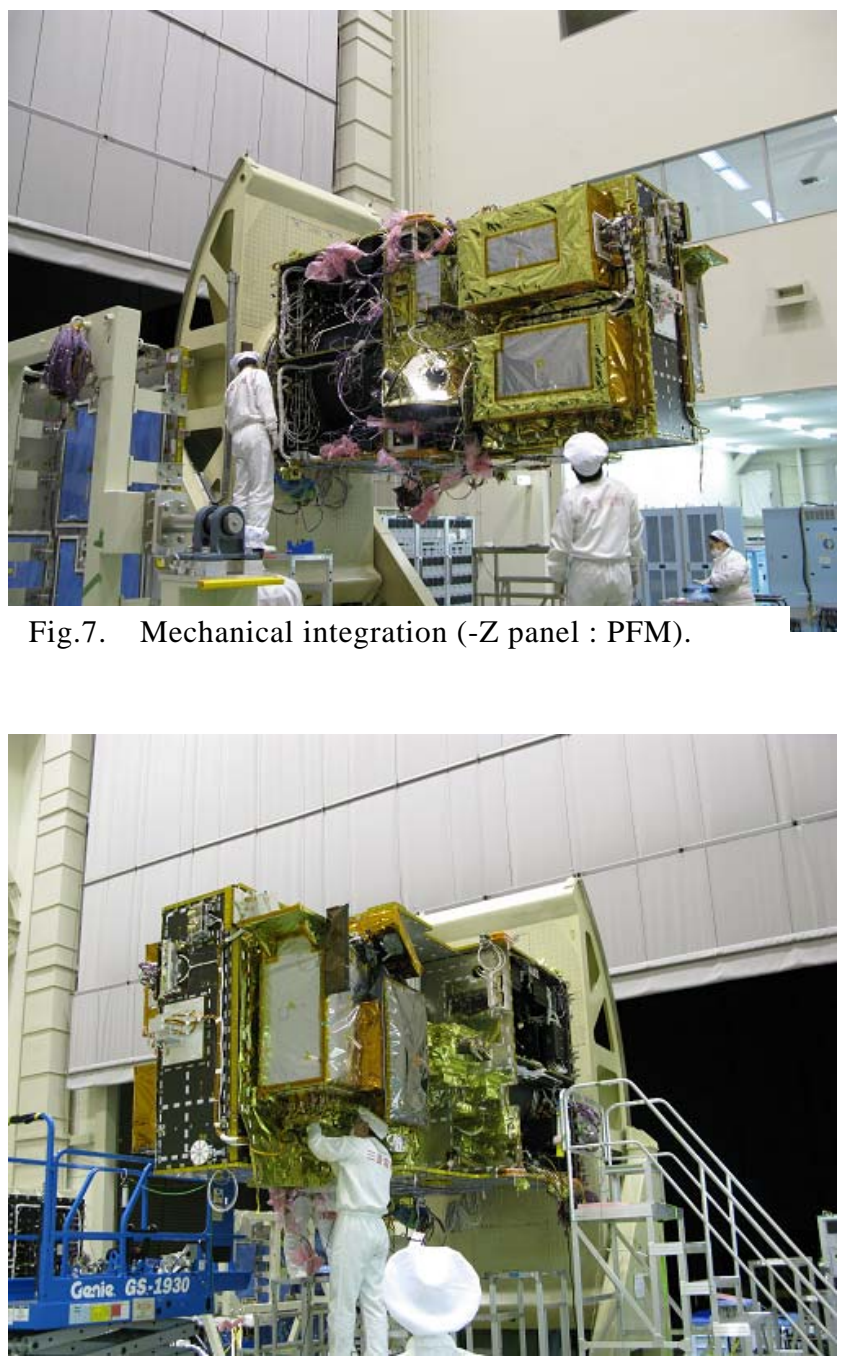

Fig.8. Mechanical integration (+Z panel : PFM).

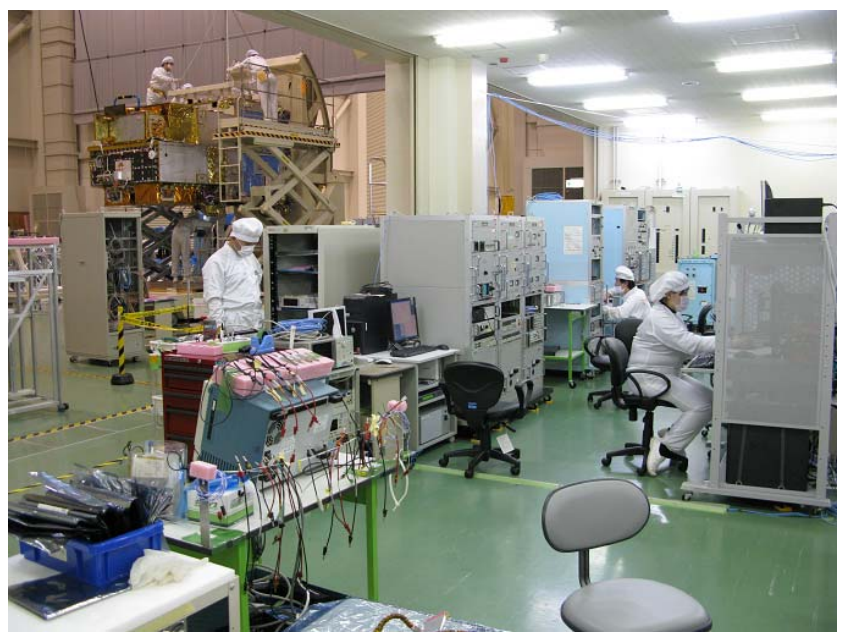

Fig.9. System initial electrical performance test (PFM). 

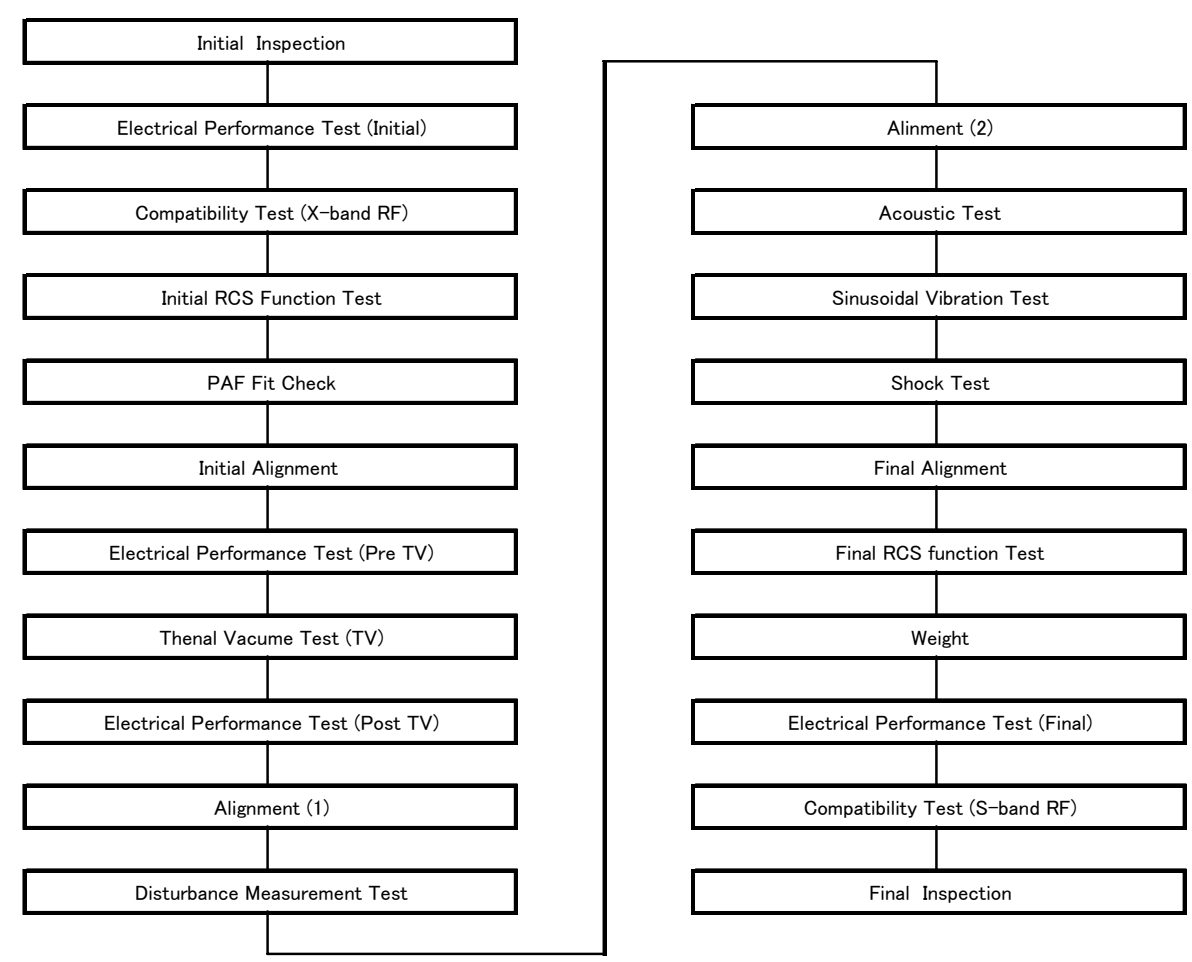

Fig.10. Flow of the system proto flight test.

Fig.10. shows the flow of the system proto flight test. At present initial inspection, electrical performance test (initial), compatibility test (X-band RF), initial RCS function test, PAF fit check and initial alignment were performed, and the following test as thermal vacuum test are being performed.

\section{Conclusion}

In this paper, the feature of the satellite bus system, subsystems and the development status of the satellite bus system were reported. The system proto flight test is in progress and the verification is being performed certainly towards its goal.

\section{References}

1) Miyazaki, H., Hasegawa, H., Komagata, F. Okada, K., Ishizu, T.: Greenhouse Gases Observing Satellite, Mitsubishi Denki Giho 79(8) (2005), pp.23-26. 